\title{
L'impact Des Normes Alimentaires Traditionnelles Sur Les Conseils Nutritionnels Chez Les Gestantes Ébrié Akouai ${ }^{72}$ De Côte d'Ivoire
}

\author{
Mathieu Hermann Coulibaly \\ Ph.D, Socio-Anthropologue, \\ Université Alassane Ouattara (UAO) de Bouaké, Côte d’Ivoire
}

doi: 10.19044/esj.2017.v13n5p481 URL:http://dx.doi.org/10.19044/esj.2017.v13n5p481

\begin{abstract}
Nutritional counseling disseminated by biomedicine during prenatal consultations is of particular importance in the context of reproductive health. These advices are given to the pregnant woman in order to prevent all forms of food imbalances that could harm her as well as the fetus. This is why it is essential for pregnant women to take this into account. But at the Ébrié akouai in Bingerville, pregnant women must also respect traditional food norms. The existence of this traditional diet is not without effects on nutritional counseling. These effects are manifold and result in noncompliance with nutritional recommendations. At the same time, they show the limits of the nutrition education of pregnant. We have tried to discover them through individual conversations, focus group and direct observations.
\end{abstract}

Keywords: Traditional food norms, nutritional counseling, food deficiencies, reproductive health, Ébrié

\section{Résumé}

Les conseils nutritionnels (CN) diffusés par la biomédecine au cours des consultations prénatales revêtent une importance particulière dans le cadre de la santé de la reproduction. Ces conseils sont donnés à la gestante en vue de prévenir toutes les formes de déséquilibres alimentaires qui pourraient lui nuire de même qu'au fœtus. C'est pourquoi il est indispensable pour les femmes enceintes d'en tenir compte. Mais chez les Ébrié akouai de Bingerville, les gestantes doivent également respecter des normes alimentaires traditionnelles (NAT). L'existence de cette diététique traditionnelle n'est pas sans effets sur les CN. Ces effets sont multiples et ont

72Orthographe la plus utilisée par la population et l'administration. Certains auteurs écrivent Kwé ou Akwè (Augé, 1375 ; Niangoran-Bouah, 1969). 
pour conséquence le non respect des recommandations nutritionnelles. Ils montrent par la même occasion les limites de l'éducation nutritionnelle des gestantes ébrié. Nous avons tenté de les découvrir à travers des entretiens individuels, des focus group et des observations directes.

Mots clés: Normes alimentaires traditionnelles, conseils nutritionnels, carence nutritionnelle, santé de la reproduction, Ébrié

\section{Introduction}

Les différentes normes alimentaires qu'elles soient traditionnelles ou nutritionnelles ont pour objectif d'aider le consommateur à faire des choix adéquats entre les aliments. Il s'agit de favoriser une alimentation qui garantisse sa santé. C'est cette préoccupation que l'on observe dans le cadre de l'alimentation des gestantes ébrié. À travers les normes alimentaires traditionnelles (NAT) et les conseils nutritionnels (CN), la société (traditionnelle ou nationale) veut les protéger de tout risque lié aux aliments. La santé est également un sujet important pour les gestantes elles-mêmes, même si elles ne se plient pas toujours aux différentes injonctions alimentaires (Coulibaly et Kouadio, 2015). La santé apparaît donc une question permanente de l'alimentation de la gestante ébrié. Cependant cet objectif de sécurité sanitaire des aliments ${ }^{73}$ est-il vraiment atteint notamment pour ce qui est des CN ? C'est l'interrogation majeure de cet article qui vise à montrer l'impact de l'existence des NAT sur la mise en pratique des $\mathrm{CN}$ en pays ébrié akouai.

Pour ce faire on présentera dans un premier temps la méthodologie. En second lieu on pourra aborder les principaux résultats et discussion.

La revue de la littérature révèle que l'éducation nutritionnelle se présente comme un processus qui privilégie l'acquisition du savoir et du savoir-faire par une approche essentiellement cognitive (Bour, 1994). De ce fait Bour pense que le manque de connaissance est à la base de mauvaises habitudes alimentaires. L'éducation nutritionnelle est donc basée sur la conviction que l'ignorance et le manque de connaissance nutritionnelle sont les principales causes de malnutrition des populations. Pourtant en dépit de nombreux programmes d'éducation nutritionnelle, on constate l'échec de la modification des pratiques alimentaires des populations notamment dans les pays en voie de développement (Adrien et Beghin, 1993). Diverses critiques permettent d'expliquer cette situation.

73L'expression, «sécurité sanitaire des aliments », se rapporte aux risques associés à la consommation des aliments (qualité de la nourriture) à la différence de la notion de « sécurité alimentaire » qui se réfère aux dispositifs mis en œuvre pour éviter le manque ou la rareté des nourritures (Poulain, 2002). 
Les nutritionnistes ont tendance à percevoir la décision alimentaire comme un acte purement individuel et rationnel. Ce postulat d'un mangeur rationnel conduit à une vision simpliste de la décision alimentaire en termes de coût-bénéfice (Cassuto, 2008). En effet, les nutritionnistes pensent que les individus qui ont la juste information seront amenés à s’y conformer. « Mais il ne suffit pas qu'une information soit considérée comme juste pour pouvoir rectifier les comportements alimentaires. " (Kaufmann, 2006, 29). La possession du savoir ne modifie donc pas forcement les «conduites à risque » (Peretti-Watel, 2001). La difficulté pour l'éducation nutritionnelle c'est celle de son adéquation avec la notion de liberté des individus (Chauliac, Masse Raimbault et De Agostino, 1991). Certes la participation des individus est une démarche indispensable, mais elle implique une prise de risque et une pleine conscience des choix à faire entre différents intérêts du point de vue de la population, des professionnels et de l'administration. Les décisions alimentaires ne sont donc pas uniquement individuelles (Cassuto, 2008). Il est de plus connu que dans toutes les sociétés traditionnelles il existe une diététique profane qui fournit un corps de connaissance dans la perspective préventive et curative (Apfelbaum et Romon, 2004). Elle définit deux systèmes classificatoires qui comprennent des catégories de mangeurs et des catégories d’aliments. Ces catégories structurent l'ordre du mangeable et permettent de penser le lien entre alimentation et santé (Corbeau et Poulain, 2002).

L’éducation nutritionnelle semble en outre favoriser le processus de médicalisation de l'alimentation (Poulain, 2009). Elle occulte par là les dimensions symboliques, imaginaires et identitaires de l'acte alimentaires (Parmentier, 2016). C'est donc une perception réductionniste du fait alimentaire car les considérations telles que le goût et le coût sont des caractères parfois plus importants que les connaissances nutritionnelles (Wilkinson, 1989). La posture des éducateurs vis-à-vis des populations cibles peut être également à l'origine des échecs des campagnes d'éducation nutritionnelle. Le détenteur du savoir occupe une position dominante (Adrien et Beghin, 1993). Il estime son savoir supérieur à celui provenant d'autres sources. La distance est accentuée par le fait que l'information est donnée à travers discours, exposés et conseils. L’échange est par conséquent négligé. Il privilégie en outre l'intervention des médias. Pourtant une relation de proximité entre émetteur et récepteur est indiquée pour que le message reçoive une adhésion des individus. Ainsi l'intervention de proches tels que les leaders d'opinion peut permettre aux messages des médias d'être incorporés et appropriés (Corbeau et Poulain, 2002). Les relations interpersonnelles qui impliquent réciprocité et proximité sont aussi gages de confiance dans le savoir des experts (Peretti-Watel, 2000). 
La multiplicité de ce savoir est souvent à l'origine d'une cacophonie diététique dans le domaine de la nutrition. L’individu est assailli par des normes contradictoires et fluctuantes (Poulain, 2008). Les savoirs partiels et provisoires sont pourtant source d’instabilité et d’incertitude (Peretti-Watel, 2001). À cela s’ajoute, notamment en zone urbaine, une multitude de canaux d’informations (mass média, réseau social...) qui entraîne une difficulté de respect des savoirs scientifiques (Ouédraogo, 1994). L’absence d’une grille d'analyse objective conduit par conséquent à des pratiques approximatives, des confusions pouvant bouleverser le modèle alimentaire (Poulain, 2008).

La réussite de l'éducation nutritionnelle est assez souvent tributaire de la situation économique des populations. La Banque Mondiale (1999) a ainsi constaté qu'il existe une forte corrélation entre les pratiques des ménages ivoiriens et les crises économiques. Celles-ci se détériorent durant ces périodes. Kouakou (1983) a ainsi observé que la récession économique entre 1980 et 1983 a entraîné dans la ville d’Abidjan la recherche de solutions appropriées d'économie et de réduction du train de vie. Les « maquis », outre leur principale clientèle issue de milieu modeste, ont vu arriver des personnes de classe moyenne en quête de restaurants aux prix abordables. Maire et Delpeuch (2000) observent qu'entre 1985 et 1993, des études répétées sur les ménages ont montré qu'en Côte d’Ivoire, le taux de retard de croissance pouvait doubler chez les enfants pendant les périodes de difficultés économiques. La dévaluation du F CFA du 12 janvier 1994 a affecté les dépenses alimentaires de toutes les catégories sociales mais plus encore des plus défavorisées. Face à l’inflation, les ménages ivoiriens ont opéré de multiples ajustements et substitutions au niveau de la qualité et de la quantité des aliments qu'ils consomment (Akindès, 1995). Ces modifications montrent bien que face aux difficultés économiques les connaissances nutritionnelles deviennent inopérantes (Wilkinson, 1989) et sont même remises en cause (Delpeuch et al., 1996).

Au total la science de la nutrition est marquée par des difficultés d’ordre conceptuel, stratégique et méthodologique. Elle est aussi liée à des contraintes économiques mais aussi culturelles dont notamment les normes alimentaires traditionnelles.

\section{Méthodologie}

\section{Sites et population}

C'est Bingerville, sous-préfecture et ancienne capitale de la Côte d'Ivoire, qui a servi de cadre à la présente étude. Elle s'est précisément déroulée dans les villages d'Akouai-Santai, d'Adjamé-Bingerville, de Brégbo, d'Adjin et d'Anan. Ces villages d'Ebrié ou Tchaman se localisent tout autour de la lagune « ébrié » et un peu plus au nord (Songon) et à l'est (Bingerville). C’est sur le territoire des Ébrié que s’est développée l'agglomération 
abidjanaise conduisant à la confrontation de leurs traditions avec la modernité.

\section{- L'enquête et l'analyse des données}

Les données qui ont servi à produire les résultats de cette étude ont été collectées à travers des entretiens individuels semi-directifs et des focus group. 44 gestantes et 25 personnes ressources ${ }^{74}$ ont été concernées par les entretiens individuels. Il s'est agi d'interroger toutes les femmes enceintes des 5 villages enquêtés sur une période de 6 mois. Les focus group ont été réalisés avec 8 mères de gestantes et 8 tradithérapeutes (matrones et guérisseuses). Ces différents instruments ont été complétés par l'observation directe des pratiques alimentaires des femmes. L’analyse des données a consisté en une analyse thématique (Vanoutrive, Derobertmasure et Friant, 2012).

\section{Résultats et discussion}

Les résultats de cette étude sont structurés autour de deux points : l'influence des NAT sur les CN (1) et les limites de l'éducation nutritionnelle des gestantes ébrié (2).

\section{L'influence des NAT sur les CN :}

Le détournement des $\mathrm{CN}$, les contradictions entre NAT et $\mathrm{CN}$, la mise à l'écart des mères et le choix des NAT par rapport aux CN permettent d’appréhender l'impact de l’existence des NAT sur les CN.

\subsection{Le détournement des $\mathrm{CN}$}

À l'origine, conseils nutritionnels donnés au cours des consultations prénatales (CPN), certaines pratiques sont entrées dans les mœurs des Ébrié akouai comme des interdits alimentaires. Il en est ainsi du respect des interdits liés à l'eau glacée et au sel. L’eau glacée est déconseillée aux gestantes par certaines sages-femmes. La raison est qu'elle rendrait le fœus gros et compliquerait de ce fait l'accouchement. Ce conseil ne fait cependant pas l'unanimité selon les sages-femmes interviewées. Quant au sel il est déconseillé parce que responsable d'œdème au pied. Autrefois il était courant que les médecins conseillent un régime sans sel aux gestantes (Lederer, 1963 ; Loffredo et Boury-Heyler, 1966). Aujourd'hui, selon les sages-femmes, ce conseil n’est plus systématique mais dépend des antécédents médicaux des gestantes. En dépit de ces réticences et précautions dans le corps médical, les gestantes se déconseillent l'eau glacée et le sel

74Les mères des gestantes, les sages-femmes, les médecins, les autorités villageoises et les tradithérapeutes ont constitué les personnes ressources. 
comme aliments à éviter. Elles le font dès que l'état de grossesse est connu et même contre l'avis médical. C'est ce que révèlent ces gestantes :

«Tu vois mon pied comme s'est enflé. Donc quand les femmes voient ça elles me disent de ne pas manger du sel. Ça fait que si je prépare la sauce, c'est sauce feuille ou la grillade. C'est ce que je peux manger. Sinon si c'est sauce seulement je ne peux pas (...) Le pied la même, d'après le docteur c'est une simple anomalie et qu'après l'accouchement ça va baisser. Donc ça diminue et ça revient (...) il m'a dit que je pouvais tout manger." (Femme, 8 mois de grossesse, Adjamé Bingerville)

«Toutefois les gens m'ont parlé seulement que de l'eau glacée [comme interdit]. Je suis allée m'informer chez la sage-femme et elle m'a dit que ce n'était pas vrai. " (Femme, 7 mois de grossesse, Adjamé Bingerville)

Ces contradictions auxquelles les gestantes sont confrontées ne sont pas sans conséquence.

\subsection{L’impact des contradictions}

Le poids des contradictions entre $\mathrm{NAT}^{75}$ et $\mathrm{CN}$ se fait ressentir au niveau des pratiques des femmes. On observe ainsi des formes de pratiques mixtes. Ces pratiques débouchent sur des situations dont les femmes ne sont pas toujours conscientes. Ainsi, certaines femmes sont convaincues de respecter les $\mathrm{CN}$. Or il n'en est rien souvent comme le montre le cas de cette enquêtée.

«On a cité la mangue, la banane douce, la pomme, le yaourt et beaucoup d'autres [comme aliments conseillés], mais on $n$ 'a pas cité ce dont on a parlé comme la papaye, l'ananas et la canne à sucre [aliments interdits].» (Femme, 8 mois de grossesse, Adjamé Bingerville)

Cette femme croit respecter à la fois normes traditionnelles et nutritionnelles. Les fruits qui lui sont interdits n’apparaissent pas concrètement dans la liste des aliments qu’on lui a conseillés à la CPN. En réalité, comme d'autres femmes dans son cas, elles ne respectent pas les CN puisque la consommation des fruits en général est vivement recommandée aux gestantes. Ces femmes sont donc perturbées par les NAT comme en témoigne l'enquêtée suivante.

"Orange, sincèrement si je bois orange ça me gêne (...) Non, on dit qu'une femme enceinte ne doit pas manger des oranges

75Pour la liste complète des aliments interdits voir Coulibaly et Gbete (2015). 
(...) On m'a dit de ne pas boire seulement. » (Femme, 4 mois de grossesse, Adjin)

Une autre enquêtée a refusé les conseils d'une sage-femme qui lui demandait de soulager une constipation récurrente en consommant de la papaye. Elle ne voulait surtout pas se mettre en porte- à -faux avec la tradition.

Ces deux femmes comme bien d'autres ne ressentent pas de malaise ou d'allergie mais seulement une gêne face à des aliments socialement marqués.

\subsection{La mise à l'écart des mères}

Dans la société ébrié, les gestantes sont encadrées au cours de leur grossesse par leur mère ${ }^{76}$. Cependant, les relations conflictuelles entre mère et fille, au sujet de l'alimentation, ont un impact sur la mise en pratique des CN. En effet, la question du respect des interdits alimentaires par les filles est un sujet source de tensions. Celles-ci se traduisent par des discussions houleuses voire des conflits. Toutefois des initiatives tendant à les éviter sont prises.

Les mères se montrent ainsi moins regardantes sur l'alimentation de leur fille et n’interviennent qu'en cas de problème. Elles se plaignent moins et observent. Pour leur part, les filles adoptent une attitude semblable. Elles impliquent peu leur mère dans leurs choix alimentaires dont les CN. Ces conseils comme noté plus haut contredisent parfois les NAT. C'est pourquoi d'ailleurs des sages-femmes demandent aux filles de transgresser certaines de ces interdits sans en parler à leurs proches. Mais le mutisme que fille et mère observent s'étend au-delà et touchent l'ensemble des questions alimentaires abordées au cours des CPN.

"Quand je vais à l'hôpital je n'informe personne sur ce qui s'y est passé. Parce que je me dis que ce qu'on me dit là c'est pour moi seule c'est pourquoi je ne dis pas ça à quelqu'un. » (Femme, 8 mois de grossesse, Akouai Santai)

"Non, je n'en [des CN] discute avec personne » (femme, 7 mois de grossesse, Adjamé Bingerville) mères :

Ces phrases qu’on entend du côté des filles sont confirmées par les

«Non, franchement ça je ne lui demande pas. Elle aussi quand elle vient, elle ne me dit pas ce qu'on lui a dit.» (Mère, Akouai Santai)

76Cet encadrement a lieu soit chez la gestante, soit au domicile de ses parents. Mais la majorité des enquêtées vivent encore chez leur parent. 
« Non. Quand elle vient elle ne me dit rien.» (Mère, Adjamé Bingerville)

Consciemment ou inconsciemment les filles excluent leur mère de ces questions. Pour leur part les mères favorisent cette attitude sans vraiment le vouloir. Certes ces conflits ne sont pas l'unique cause de cette absence de communication, mais ils l'entretiennent. L'alimentation est en effet un sujet polémique entre mère et fille. De plus les stratégies adoptées par les unes et les autres pour éviter les querelles inhérentes à ce sujet aboutissent à la mise à l'écart des mères. Ces dernières qui personnalisent les pratiques traditionnelles sont ainsi discréditées. La conséquence de cette exclusion est importante.

Les mères sont des "gatekeepers » (Lewin, 1943), c'est-à-dire les portiers qui contrôlent l'accès du « canal » par lequel l'aliment arrive dans le ménage. En ne les faisant pas participer aux CPN, les filles se mettent en difficulté notamment pour ce qui est du respect des CN. Pour les filles la complexité du problème se résume dans cette question : comment intégrer les aliments conseillés dans le régime alimentaire familial ou personnel sans évoquer la question de l'alimentation avec leur mère ? Plus concrètement, comment emmener sa mère à cuisiner par exemple les sauces à base de feuille sans discuter avec elle des $\mathrm{CN}$ ?

La réponse n'est pas évidente. Il n'est pas étonnant que des filles disent ne pas consommer souvent des sauces à base de feuille parce qu'elles n'en ont pas l'habitude. Si la famille a depuis toujours l'habitude d'en manger, il n'y a pas de problème. Mais le contraire est problématique car il faut alors sensibiliser la mère. Effectivement, si elle n'est pas au fait des recommandations données par les sages-femmes, elle ne peut pas connaître l'importance de ces sauces et de bien d'autres questions du même niveau. Une mère, qui ne sait pas par exemple que les fruits sont indispensables à sa fille, ne peut pas les lui offrir régulièrement. Or bien souvent les filles dépendent financièrement de leur mère.

Un constat est à faire. Le rôle de la mère se trouve fragilisé par cette situation. La mère n'est plus seule à orienter sa fille sur les questions alimentaires. Elle en est même mise en marge. Au-delà de la mère c'est l'institution traditionnelle de prise en charge des gestantes qui s'en trouve fragilisée. Mais cette situation rejaillit sur le suivi des CN. En effet, écarter les mères c'est se priver du soutien capable d'apporter les moyens d'améliorer son alimentation. Les CN sont de ce fait tributaires des NAT. Pour éviter des polémiques à leur sujet, les gestantes s'exposent à des risques de déficits nutritionnels. Pourtant l'implication des mères peut être une chose positive.

La mère d'une enquêtée du village d'Anan montre qu'il est possible de les y associer. En effet, elle veille à ce que sa fille respecte les NAT. C'est 
pourquoi elle lui en a parlé. Mais elle ne se met pas pour autant en marge des CPN.

"Oui. Elle dit qu'elle sait. Elle accompagne sa fille. Là-bas

[à la maternité], on leur présente des brochures, des images sur lesquels il y a ce qu'il faut faire... » [Interprète]

C'est pourquoi elle aide sa fille en lui achetant ce qu'on lui a conseillé de consommer.

"Elle suit à la lettre. Elle la conseille... seulement les nuits, elle ne lui conseille pas de manger assez. Quand elle revient de Bingerville, elle lui apporte la carotte, la salade, des fruits. Elle suit ça. De plus tout ce qui est interdit [par la tradition], elle demande à sa fille de ne pas en manger. Elle respecte cela aussi. » [Interprète]

On peut reprocher à cette mère d'insister encore sur les interdits, mais il faut remarquer qu'elle n'abandonne pas sa fille en ce qui concerne les CN.

\subsection{Le choix des NAT face aux $\mathrm{CN}$}

Face aux CN, les gestantes choisissent parfois de se référer aux NAT. Certaines femmes disent croire aux interdits et prescriptions que les NAT préconisent. Elles n'y ont pas forcément cru avant. Mais elles s'y plient à cause des représentations symboliques qui pèsent sur les aliments. En effet, les conséquences liées aux aliments interdits les conduisent au respect des NAT. D'autres les ont adopté parce qu'elles affirment avoir été victimes de leur non respect ou connaître des proches qui l'ont été. L'implication de leurs proches est aussi un facteur déterminant. Ceux-ci veillent au respect des NAT. Même si ce n'est pas le cas, certaines affirment les respecter par peur d'être indexées comme responsables de tout problème qui pourrait subvenir. Certaines de ces femmes sont issues de familles où les NAT ne sont pas connues ou imposées, mais elles ont décidé de les respecter. Elles ont été conseillées dans ce sens par des amies ou des personnes âgées en qui elles ont confiance. Pour toutes ces raisons l'ensemble de ces femmes qui respectent les NAT au départ ne se conforment pas aux CN surtout quand ils se contredisent.

Malgré leur participation aux CPN, certaines gestantes ne se conforment pas aux CN. Pour ces gestantes, les $\mathrm{CN}$ ne répondent pas toujours à leurs besoins. Les femmes qui ont choisi de se faire suivre dans les établissements sanitaires peuvent se retrouver ainsi devant une impasse (Coulibaly et Gbete, 2015). Elles ne trouvent pas toujours de réponses à leur problème de santé. Cette situation vient du fait qu'elles sont parfois embarrassées à parler des effets de certains interdits alimentaires aux agents de santé. Ce sont pour elles des croyances qui peuvent provoquer de l'incompréhension pire de la moquerie. Il y a aussi le fait que les gestantes 
jugent la biomédecine inefficace pour traiter certaines maladies comme la maladie hémorroïdaire et la maladie de la fontanelle. S'ajoutent aussi les maladies comme les taches de rousseur sur la peau des enfants dont la biomédecine semble avoir du mal à déterminer les causes. Pour toutes ces raisons, elles sont emmenées à se replier sur les NAT qui leur donnent des réponses à travers les interdits alimentaires.

Tous ces faits ne permettent pas aux gestantes d'adopter pleinement les CN. Ils les perturbent et présentent les NAT comme une alternative crédible. Au final, ils mettent en évidence les difficultés de l'éducation nutritionnelle des gestantes ébrié.

\section{Les limites de l'éducation nutritionnelle des gestantes ébrié akouai}

Deux principales raisons expliquent les limites de l'éducation nutritionnelle des gestantes ébrié à savoir leur conception de la maladie et la solidarité qu'elles trouvent entre l'éducation nutritionnelle et les autres spécialités biomédicales.

\subsection{La conception traditionnelle de la maladie et de la santé}

À la différence de la biomédecine, « les autres façons de concevoir la maladie laissent une place à la personne, au monde, au surnaturel dans leur identification du mal, qu'il s'agisse de son siège, de ses causes ou de son destin » (Benoist, 2004, 10). Ainsi la représentation de la santé et de la maladie par les ébrié souligne les limites de l'éducation nutritionnelle. Pour Memel-Fotê (1998), la maladie est pour les Ivoiriens un état physique, psychologique et social de l'individu. Les interdits alimentaires chez les Ébrié montrent que l'alimentation recouvre cette réalité. Les interdits alimentaires en effet ne conduisent pas uniquement qu'à des conséquences d'ordre physique. Ils englobent aussi des conséquences d'ordre social et psychologique. L'aliment va donc au-delà du simple biologique. Or la nutrition ne s'intéresse qu'aux effets des aliments et aux troubles physiques qui en découlent. Elle ne s'intéresse qu'à la dimension santé de l'aliment occultant ses dimensions hédonique, sociale et symbolique. Elle ne peut donc pas prémunir contre les troubles sociaux et psychologiques que les femmes ébrié akouai identifient dans leur rapport à l'alimentation. Ce sont pourtant des préoccupations importantes pour ces femmes. De ce fait, la nutrition et la biomédecine en général ne comblent pas toujours leurs attentes. Il n'est par conséquent pas aisé pour les agents de santé à travers les CN de détourner ces femmes des NAT.

\subsection{La solidarité entre les spécialités médicales}

La seconde raison est celle liée au fait que l'éducation nutritionnelle des gestantes est étroitement liée à d’autres spécialités médicales. En effet, 
plusieurs branches médicales encadrent la gestante, la nourrisse et l'enfant. Il s'agit particulièrement de la gynécologie, de l'obstétrique, de la médecine générale, de la pédiatrie et de la nutrition. Pour les populations enquêtées, il existe une solidarité entre toutes ces spécialités et la nutrition. Ainsi le succès ou l'échec de l'une d'entre elles influence celui de l'éducation nutritionnelle des gestantes. Les CN sont donc acceptés et jugés par les femmes ébrié à l'aune de leurs expériences avec les autres spécialités de la médecine. Ainsi quand elles ne trouvent pas de réponses favorables à leurs inquiétudes, cela rejailli sur le suivi des CN. La diffusion des CN par les agents de santé chargés des CPN et non par des nutritionnistes ou agents spécialisés contribue également à une telle réalité. Les relations difficiles que les gestantes ébrié ont avec les sages-femmes entraînent ainsi une dépréciation des activités de ces dernières et par conséquent des CN. Les échecs des soins du pédiatre et du généraliste, entraînent également un refus des $\mathrm{CN}$ par les femmes.

\section{Conclusion}

Cet article a permis de souligner les différents impacts qu'ont les NAT sur les CN chez les Ébrié. Les NAT conduisent au détournement de certains $\mathrm{CN}$ en les intégrant. Elles sont également sources de contradictions dont l'effet est de conduire les gestantes à des pratiques contraires aux recommandations nutritionnelles. Elles conduisent aussi à la mise à l'écart des mères dans la prise en charge biomédicale des gestantes. Ce fait est préjudiciable à ces dernières puisque les mères peuvent garantir un suivi adéquat des CN. Il y a enfin l'impasse devant laquelle se trouvent les femmes qui ont fait confiance aux structures de santé. Elles ne trouvent pas toujours de satisfaction avec celles-ci et doivent se replier sur les NAT avec les risques que cela comporte. Ces impacts mettent en lumière les limites de l'éducation nutritionnelle des gestantes ébrié. Elles sont liées à une approche réductrice de l'aliment par la nutrition qui rend cette dernière inefficace aux yeux des femmes ébrié. Elles tiennent aussi au lien étroit entre les spécialités médicales qui conduit à une remise en cause des CN. Dans ce contexte, comment faire en sorte que la science de la nutrition à travers les $\mathrm{CN}$ puisse encadrer efficacement les gestantes ébrié akouai et plus généralement les populations dotées de NAT?

\section{References:}

1. Adrien, M. et Beghin, I. (1993). Communication et nutrition. De l'éducation nutritionnelle à la communication sociale en nutrition. Paris : L'Harmattan, $157 \mathrm{p}$.

2. Akindès, F. (1995). Dévaluation et alimentation à Abidjan (Côte d'Ivoire). Les cahiers de la Recherche Développement, (40), 24-42. 
3. Apfelbaum, M. et Romon M. (2004). Diététique et nutrition (6e éd.). Paris : Masson, 128 p.

4. Augé, M. (1975). Théories des pouvoirs et idéologie : étude de cas en Côte d'Ivoire. Paris : Hermann, 440 p.

5. Benoist, J (2004). « Rencontres de médecines: s'opposer ou s'ajuster ». L'autre, Cliniques, cultures et sociétés, 5 (2), 277-286.

6. Bour, H. (1994). L'éducation nutritionnelle. Réflexions et méthodologie. Cahiers de nutrition et de diététique, 29 (2), 66-67.

7. Cassuto, D.A. (2008). Avant les réponses, les questions ! Dans Actes de colloque, Eduquer les mangeurs ? De l'éducation nutritionnelle à l'éducation alimentaire du 9 décembre 2008. Récupéré du site http://www.alimentation-sante.org

8. Chauliac, M., Masse Raimbault, A. M. et De Agostino, M. (1991). L'Éducation nutritionnelle. L'Enfant en milieu tropical, (192), 7-9.

9. Corbeau, J.-P. et Poulain J.-P. (2002). Penser l'alimentation. Toulouse : Privat/Ocha, 209 p.

10. Coulibaly, M. H. et Gbete, I.J-M. (2015). La régulation des interdits alimentaires liés à la grossesse et ses implications pour la santé des femmes ébrié (Côte d'Ivoire). Geste et Voix, (22), 275-299.

11. Coulibaly, M. H. et Kouadio, K. M. D. (2015). L'incertitude, ressource de l'action face aux interdits alimentaires liés aux grossesses chez les Ébrié de Côte d'Ivoire. Lettres D'Ivoire, (21), 121-127.

12. Delpeuch, F., Martin-Prével, Y, Fouéré, T, Traissac, P., Mbemba, F., Ly, C.,... Maire, B. (1996). L’alimentation de complément du jeune enfant après la dévaluation du franc CFA : deux études de cas en milieu urbain, au Congo et au Sénégal. Bulletin de l'Organisation mondial de la Santé, 74 (1), 67-75.

13. Kaufmann, J-C. (2006). Casseroles, amour et crises. Ce que cuisiner veut dire. Paris : Hachette Littérature, 342 p.

14. Kouakou, N. F. (1983). Les “'maquis”' d'Abidjan : nourritures du terroir et fraternité citadine ou la conscience de classe autour d'un foutou d'igname. Cahiers ORSTOM, XIX (4), 545-550.

15. Banque Mondiale (1999). État nutritionnel et la pauvreté en Afrique Subsaharienne. Récupéré du site www.imnrc.org

16. Lederer, J. (1963). Diététique du médecin praticien (2ème éd.). Paris : Masson et Cie Editeurs, 591 p.

17. Lewin, K. (1943). Forces behind foods habits and methods of changes. Bulletin of the National Research Council, (108, October), 35-65. 
18. Loffredo, V. et Boury-Heyler, C. (1966). Précis d'obstétrique. À l'usage des infirmières et professions de paramédicales (2ème éd.). Paris : Lamarre Poinat, 142 p.

19. Maire, B. et Delpeuch, F. (2000). Nutrition et alimentation en Afrique au sud du Sahara, les défis du XXIe siècle. Afrique contemporaine, (195), 136-154.

20. Memel-Fotê, H. (1998). Les représentations sociales de la santé et de la maladie chez les Ivoiriens. Paris : L’Harmattan, 209 p.

21. Niangoran-Bouah, G. (1969). Les Ébrié et leur organisation politique traditionnelle. Annales de l'Université d'Abidjan, (Série F, Tome.1, fascicule 1), 51-89.

22. Ouédraogo, C. (1994). Éducation de la femme et soins aux enfants à Ouagadougou, (27), Paris : Les dossiers du CEPED, 39 p.

23. Parmentier, I. (2016). Prévention: "Diététique et information nutritionnelle: améliorer l'information et l'éducation nutritionnelle ». Récupéré du site www.uiparm.fr

24. Peretti-Watel, P. (2000). Sociologie du risque. Paris : Armand Colin, $286 \mathrm{p}$.

25. Peretti-Watel, P. (2001). La société du risque. Paris : La découverte et Syros, 126 p.

26. Poulain, J-P. (2002). Sociologies de l'alimentation. Paris : PUF, 286 p.

27. Poulain, J-P. (2008). Manger aujourd'hui : attitudes, normes et pratiques. Toulouse : Editions Privat, 235 p.

28. Poulain, J-P. (2009). Sociologie de l'obésité. Paris: Presses Universitaires de France, 360 p.

29. Vanoutrive, J., Derobertmasure, A. et Friant, N. (2012). Analyse thématique et analyse propositionnelle : application `a un corpus de témoignages concernant l'injustice scolaire. Mesure et évaluation en éducation, 35 (2), 97-123.

30. Wilkinson, R.G. (1989). Research methods in nutritional anthropology. The United Nation University, 217 p. 